\title{
CRECIMIENTO Y EFICIENCIA FOTOQUÍMICA DEL FOTOSIS- TEMA II EN PLANTAS DE FRESA (Fragaria sp.) AFECTADAS POR LA CALIDAD DE LA LUZ: IMPLICACIONES AGRONÓMICAS
}

\author{
GROWTH AND PHOTOCHEMICAL EFFICIENCY OF \\ PHOTOSYSTEM II IN STRAWBERRY PLANTS (Fragaria \\ sp.) AFFECTED BY THE LIGHT QUALITY: AGRONOMIC \\ IMPLICATIONS
}

\author{
Fánor Casierra-Posada1, Jaime E. Peña-Olmos¹, Christian Ulrichs²
}

\begin{abstract}
1 Universidad Pedagógica y Tecnológica de Colombia (UPTC), Facultad de Ciencias Agropecuarias, Grupo de Investigación Ecofisiología Vegetal. Avenida Central del Norte, Tunja, Colombia. Autor para correspondencia: fanor.casierra@uptc.edu.co ${ }^{2} \mathrm{Humboldt}$ Universität zu Berlin, Faculty for Agriculture and Horticulture, Division Urban Plant Ecophysiology, Lentzeallee 55/57, 14195 Berlin, Germany.
\end{abstract}

Rev. U.D.C.A Act. E Div. Cient. 14(2): 43 - 53, 2011

\section{RESUMEN}

Fotomorfogénesis es cualquier cambio de forma o de función en un organismo, que sucede en respuesta a las modificaciones en la luz ambiental. La calidad de la luz, se capta mediante receptores específicos para las diferentes longitudes de onda. En un ensayo factorial, se evaluó la influencia de la calidad de la luz sobre el crecimiento y la eficiencia fotoquímica del fotosistema II (PSII) en plantas de fresa (Fragaria sp., cv. Chandler), bajo invernadero, en Tunja, Colombia. Las plantas crecieron bajo diferente calidad de luz (amarilla, verde, azul, roja, transparente y el control sin cobertura), proporcionada por películas filtro de polipropileno. Se realizaron determinaciones de florescencia mínima $\left(F_{0}\right)$, máxima $\left(F_{m}\right)$, variable $\left(F_{v}\right)$, terminal $\left(F_{t}\right)$ y la eficiencia fotoquímica máxima $\left(\mathrm{F}_{\mathrm{v}} / \mathrm{F}_{\mathrm{m}}\right)$, en hojas adaptadas a la oscuridad, 32 semanas luego del trasplante. En relación con el crecimiento, las plantas control registraron los valores más altos de materia seca por planta y de peso específico de las hojas. Las plantas bajo el filtro rojo desarrollaron la mayor área foliar, mientras que el filtro verde redujo el crecimiento. Las películas de color modificaron el patrón de asignación de materia seca en los órganos de las plantas, especialmente, el filtro de color verde. La luz filtrada afectó todos los parámetros de la fluorescencia de la clorofila. En relación con las plantas control $F_{v}, F_{m}$ y $F_{t}$ fueron mayores en las plantas que crecieron bajo todas las coberturas. Mientras que $F_{0}$ fue mayor bajo las coberturas amarilla y transparente, $F_{v} / F_{m}$ fue menor bajo esas películas filtro. Los resultados se discuten en relación con sus posibles implicaciones sobre prácticas agronómicas apropiadas.

Palabras clave: Fotomorfogénesis, material seca, partición de biomasa, luz coloreada, $\mathrm{F}_{\mathrm{v}} / \mathrm{F}_{\mathrm{m}}$, fluorescencia mínima, fluorescencia máxima.

\section{SUMMARY}

Photomorphogenesis is any change in form or function of an organism occurring in response to changes in the light environment. Light quality is mainly sensed by the presence of different light receptors specific for different wavelengths. The influence of light quality on the growth and photochemical efficiency of photosystem II (PSII) in strawberry plants (Fragaria sp., cv. Chandler) was examined in a factorial set up, under greenhouse in Tunja / Colombia. Plants grew under diverse light qualities (yellow, green, blue, red, transparent, and control without colored cover) provided by polypropylene filter films. Determinations of minimum $\left(\mathrm{F}_{0}\right)$, maximum $\left(\mathrm{F}_{\mathrm{m}}\right)$, variable $\left(F_{v}\right)$, and terminal $\left(F_{t}\right)$ fluorescence and maximum photochemical efficiency $\left(\mathrm{F}_{\mathrm{v}} / \mathrm{F}_{\mathrm{m}}\right)$ of dark-adapted leaves were done 32 weeks after the transplant. Related to growth, 
control plants recorded highest dry matter and specific leaf weight. Plants growing under the red filter developed highest leaf area. The green film reduced the growth. The color films changed the dry matter partitioning patterns in the plants organs, specially the green filter. Filtered light affected the chlorophyll fluorescence variables, related to control plants. Regards to control plants, $F_{v}, F_{m}$ and $F_{t}$ were higher in plants growing under all covers. While $\mathrm{F}_{0}$ was higher under the transparent and yellow films, $F_{v} / F_{m}$ was lower under these covers. The results were discussed in relation to possible implications for appropriate agronomic practices.

Key words: Photomorphogenesis, dry matter, biomass partitioning, colored light, $F_{v} / F_{m}$, minimal fluorescence, maximal fluorescence.

\section{INTRODUCCIÓN}

Los vegetales poseen mecanismos fotoselectivos sofisticados para capturar la energía lumínica, necesaria para la fotosíntesis (Jiao et al. 2007). La intensidad y la calidad de la luz son factores determinantes en el crecimiento y desarrollo de los vegetales (Fukuda et al. 2008). Los cambios en la calidad de la luz afectan considerablemente muchos parámetros anatómicos, fisiológicos, morfológicos y bioquímicos en las plantas (Haliapas et al. 2008).

La fotomorfogénesis involucra tres grupos importantes de fotorreceptores transductores de información: los fitocromos, los receptores de la luz azul y los fotorreceptores de luz UV-B (Jiao et al. 2007), aunque también se ha reportado la presencia de un compuesto a base de zeaxantina como receptor de luz verde (Folta \& Maruhnich, 2007). El rango máximo de absorción de la forma Pr del fitocromo asemeja el de las clorofilas (rojo); sin embargo, la forma Pfr absorbe a longitudes de onda más largas (rojo lejano) (Mathews, 2010). Junto con los fitocromos, se encuentran otros receptores de la luz azul, como los criptocromos y las fototropinas (Strasser et al. 2010). Los fotorreceptores UV-B están también implicados en algunas respuestas fotomorfogénicas controladas por las fototropinas. En la última década, se caracterizó un fotorreceptor de luz azul denominado 'zeitlupe' (Imaizumi et al. 2003).

El efecto de la calidad de la luz ha sido motivo de investigación, con el objeto de sacar provecho del efecto fotomorfogénico inducido en los vegetales por las diferentes longitudes de onda de la luz incidente (Shahak et al. 2008; Casierra-Posada $\&$ Rojas, 2009). En las últimas tres décadas, el efecto de la calidad de luz sobre algunas hortalizas y frutales se ha estudiado con la utilización de láminas coloreadas sobre el suelo (mulch plástico), las cuales, reflejan la luz de diferentes longitudes de onda, que cambian el microclima alrededor de las plantas (Decoteau, 2008). Algunas características de las plantas, como la tasa de crecimiento, el desarrollo de órganos vegetativos y reproductivos, la biomasa total y la distribución de foto-asimilados, fueron afectadas por calidad de la luz, particularmente, por la relación $\mathrm{r} / \mathrm{fr}$ (red/ far red) (Bradburne et al. 1989). En estudios de campo, bajo riego, la calidad de frutas de fresa en los cultivares Chandler y Sweet Charlie, en términos de concentración de compuestos aromáticos, fue superior cuando el suelo se cubrió con plástico rojo, en comparación con plástico de color negro (Loughrin \& Kasperbauer, 2002). Estos autores concluyeron que las bandas de luz en los rangos rojo y rojo lejano reflejadas desde el plástico rojo, posiblemente, actuaron a través de los sistemas de fitocromos, modificando la expresión de algunos genes. En consecuencia, aumentaron las concentraciones de compuestos aromáticos en frutos de fresa.

Miranda \& Williams (2007) evaluaron la eficiencia fotoquímica del fotosistema II (PSII) in vitro en hojas de fresa y encontraron un incremento de este parámetro, en un rango de 0,64, bajo luz blanca, a 0,80, bajo luz amarilla. Además, las hojas desarrolladas bajo luz azul fueron similares al control con luz blanca en muchos parámetros relacionados con la fluorescencia de la clorofila, con excepción del nivel inicial de la fluorescencia. Por su parte, Casierra-Posada $\&$ PeñaOlmos (2011) expusieron plantas de fresa a coberturas plásticas de diversos colores y hallaron que las diferentes calidades de luz influyeron sobre el contenido de clorofila. Además, la concentración de clorofila a fue mayor en hojas desarrolladas bajo luz de color verde y rojo, seguida de la encontrada en plantas expuestas a la cobertura de color azul, transparente y amarilla.

Asumiendo que las diferentes bandas del espectro de radiación pueden influenciar el transporte de electrones y los demás parámetros relacionados con la fluorescencia de la clorofila en plantas de fresa, se evaluó el efecto de la calidad espectral de la luz sobre la eficiencia fotoquímica del fotosistema II, bajo condiciones de invernadero en el trópico alto.

\section{MATERIALES Y MÉTODOS}

El ensayo, se desarrolló en la ciudad de Tunja, Departamento de Boyacá, en Colombia $\left(5^{\circ} 33^{\prime} 16.25^{\prime \prime} \mathrm{N} 73^{\circ} 21^{\prime} 9.14^{\prime \prime} \mathrm{O}\right.$; $2.691 \mathrm{msnm}$ ), bajo condiciones de invernadero de vidrio transparente, en el cual, diez plantas por tratamiento se sometieron a la exposición de la radiación solar, filtrada a través de películas de polipropileno de colores rojo, amarillo, azul y transparente, de un grosor de $15 \mu$. Las plantas control, se desarrollaron dentro del invernadero, sin ningún tipo de cobertura de polipropileno. La radiación fotosintéticamente 
activa (PAR) y la disminución de la luz (opacidad) registrada bajo las coberturas, se muestra en la tabla 1. De igual manera, la transmitancia de los diferentes materiales utilizados en el ensayo, se presentan en la figura 1. A pesar que el vidrio es traslúcido, este tipo de invernadero ofrecía a las plantas condiciones de alto sombreamiento (Tabla 1). Como material vegetal inicial, se tomaron estolones (importadas desde Chile) de fresa cultivar 'Chandler', que se expusieron previamente a una temperatura de $4 \pm 1^{\circ} \mathrm{C}$, durante tres semanas, con la intención de romper la dormancia de las yemas y lograr una brotación rápida y uniforme; posteriormente, los estolones se colocaron en una solución nutritiva, con la siguiente composición en mg L-1: nitrógeno nítrico, 40,3; nitrógeno amoniacal, 4,0; fósforo, 20,4; potasio, 50,6; calcio, 28,8; magnesio, 11,4; azufre, 1,0; hierro, 1,12; manganeso, 0,112; cobre, 0,012; zinc, 0,0264; boro, 0,106; molibdeno, 0,0012 y cobalto, 0,00036. Estas concentraciones corresponden a dosis utilizadas frecuentemente para cultivos hidropónicos. Se realizaron dos aplicaciones con base en Captan, para prevenir la aparición de problemas fitopatológicos, especialmente Mycosphaerella fragariae.

Tabla 1. Características de radiación fotosintéticamente activa y opacidad registradas bajo las coberturas de polipropileno de colores.

\begin{tabular}{|l|c|c|}
\hline \multirow{2}{*}{ Color de la cobertura } & $\begin{array}{c}\text { Radiación fotosintéticamente } \\
\text { activa (PAR) }\end{array}$ & Opacidad \\
\cline { 2 - 3 } & $\left(\mu \mathrm{mol} \mathrm{m}^{-2} \mathrm{~s}^{-1}\right)$ & $(\%)$ \\
\hline Amarillo & 152,19 & 49,25 \\
\hline Azul & 99,31 & 66,89 \\
\hline Rojo & 86,95 & 71,01 \\
\hline Verde & 78,87 & 73,70 \\
\hline Transparente & 210,46 & 29,83 \\
\hline Control & 299,94 & 0,00 \\
\hline
\end{tabular}

A las 32 semanas del trasplante, se midió la fluorescencia de la clorofila en hojas completamente expandidas del tercio medio de la corona, con un pulso actínico de luz de $25 \mu \mathrm{mol}$ $\mathrm{m}^{-2} \mathrm{~s}^{-1}$, mediante el uso de un fluorómetro Junior-PAM (Walz GmbH, Effeltrich, Germany). Previo a las mediciones, las hojas se expusieron a la oscuridad con pinzas de exclusión de luz. Se realizaron las siguientes mediciones: fluorescencia mínima $\left(\mathrm{F}_{0}\right)$ y fluorescencia máxima $\left(\mathrm{F}_{\mathrm{m}}\right)$. Con el uso de estos parámetros, se calculó la eficiencia fotoquímica del PSII: $\mathrm{F}_{\mathrm{v}} / \mathrm{F}_{\mathrm{m}}=\left(\mathrm{F}_{\mathrm{m}}-\mathrm{F}_{0}\right) / \mathrm{F}_{\mathrm{m}}$, y la fluorescencia variable: $\mathrm{F}_{\mathrm{v}}=\mathrm{F}_{\mathrm{m}}-\mathrm{F}_{0}$. Estas fórmulas fueron calculadas para el análisis cuantitativo de la reacción de producción de fluorescencia, acorde con la curva llamada "cinética de Kautsky" (González et al. 2008).

Se empleó un diseño en bloques al azar, teniendo el color de la cobertura como factor de bloque. Cada tratamiento constaba de diez plantas y se tomó una planta como replicación. En cada planta, se dispuso de una hoja, para un total de 10 mediciones por tratamiento. Los datos obtenidos, se sometieron a un análisis clásico de variancia $(\mathrm{P}<0,05)$ y a la prueba de separación de promedios de Tukey, mediante la aplicación PASW (Predictive Analytics Software) versión 18.0.0 (30-07-2009; IBM Corporation, Somers - USA).

\section{RESULTADOS Y DISCUSIÓN}

Las películas de polipropileno, llamadas comúnmente, papel celofán, son bastante selectivas en cuanto a las longitudes de onda que absorben y, por consecuencia, las que transmiten. Efectivamente, el polipropileno azul transfiere mayormente longitudes de onda entre los 440 y 520nm, que corresponden al espectro de color azul y verde. La película de color rojo transmite, en muy poca cantidad luz azul, pero en mayor cantidad, por encima de $610 \mathrm{~nm}$, donde se encuentra parte del espectro del rojo y rojo lejano. La película de color verde emite en mayor cantidad de longitudes de onda que se encuentran en el espectro del color verde y el color amarillo (510-540 nm). Por su parte, el polipropileno de color amarillo transmite las longitudes de onda superiores a 530nm, que corresponden al espectro en el rango de los colores verde, amarillo, naranja, rojo y rojo lejano. En cambio, bajo el papel transparente no se produce una selección del espectro y se 
transmite, en igual medida, todas las longitudes, entre los 400 y 700nm. Llama la atención, que acorde con la figura 1, el rango máximo de transmitancia de la cobertura de color amarillo se encuentra muy cercano al valor registrado para la cobertura transparente, por tanto, muchas de las variables analizadas presentan tendencias similares en plantas colocadas bajo estas dos coberturas, en especial, los valores de $\mathrm{F}_{0}, \mathrm{~F}_{\mathrm{v}} / \mathrm{F}_{\mathrm{m}}$ y $\mathrm{F}_{\mathrm{t}}$.
Crecimiento vegetal: Se registró diferencia estadísticamente significativa en el peso seco total por planta, el área foliar, el peso específico de las hojas (peso seco por área foliar) y la relación raíz:vástago $(\mathrm{P}<0,01)$ (Tabla 2$)$. En cuanto al primer parámetro, las plantas que crecieron bajo las coberturas de colores amarillo, azul y verde fueron estadísticamente diferentes a las del control y presentaron valores de peso seco 14,$52 ; 45,89$ y $84,40 \%$, respectivamente, por debajo del control. El área foliar mostró valores estadísticamente difer-

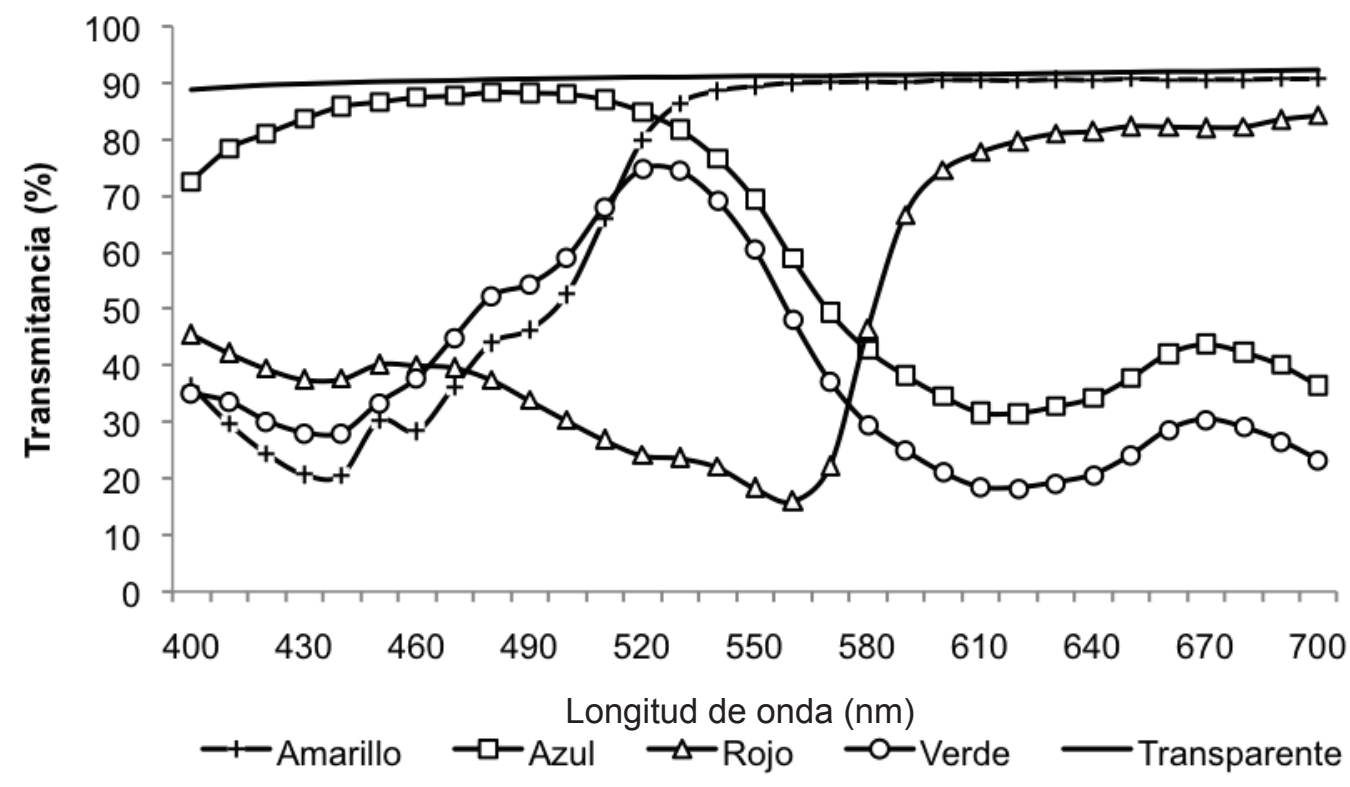

Figura 1. Transmitancia de las películas de polipropileno utilizadas como cobertura en plantas de fresa (Fragaria sp.).

Tabla 2. Parámetros de crecimiento determinados en plantas de fresa (Fragaria sp.) expuestas a coberturas de color (datos por planta).

\begin{tabular}{|c|c|c|c|c|}
\hline \multirow{2}{*}{$\begin{array}{l}\text { Color de la } \\
\text { cobertura }\end{array}$} & Peso seco total & Area foliar & Peso específico de las hojas & Relación raíz:vástago \\
\hline & (g) & $\left(\mathrm{cm}^{2}\right)$ & $(\mathrm{mg} \mathrm{cm}-2)$ & \\
\hline Amarillo & $34,69 \mathrm{~b}$ & $776,89 \quad b$ & $8,63 \mathrm{~b}$ & $0,15 \mathrm{~b}$ \\
\hline Azul & $21,96 \quad c$ & $768,73 \mathrm{~b}$ & $7,14 \mathrm{~cd}$ & $0,15 \mathrm{~b}$ \\
\hline Rojo & $36,44 a b$ & 928,37 a & 8,25 bc & $0,16 \mathrm{~b}$ \\
\hline Verde & $6,33 \mathrm{~d}$ & $348,22 \mathrm{~d}$ & $6,40 \mathrm{~d}$ & 0,39 a \\
\hline Transparente & $37,59 a b$ & $487,26 \mathrm{c}$ & 12,14 a & $0,18 \mathrm{~b}$ \\
\hline Control & 40,58 a & $527,14 \quad c$ & 12,12 a & $0,20 \mathrm{~b}$ \\
\hline
\end{tabular}


entes entre el control y las plantas colocadas bajo las coberturas amarilla, azul, roja y verde. Las tres primeras coberturas, respectivamente, presentaron valores de área foliar 47,$37 ; 45,83$ y $76,11 \%$, superiores a los valores del control, mientras que la cobertura de color verde mostró un área foliar, 33,94\%, por debajo. El valor del peso específico de las hojas, se redujo en las plantas expuestas a las coberturas de colores amarillo, azul, rojo y verde, cuyo valor promedio, respectivamente, fue 28,75; 41,09; 31,89 y 47,19\%, inferior al encontrado en las del control sin cobertura. Por último, el valor promedio de la relación raíz:vástago sólo fue estadísticamente diferente entre las plantas expuestas a la cobertura verde y las que crecieron sin cobertura; las primeras presentaron un valor $92,88 \%$ superior a la segundas, en cuanto a esta relación alométrica (Tabla 2).

De manera usual, el incremento del área foliar es una estrategia para aumentar la superficie fotosintéticamente activa, así se mejora la eficiencia en los procesos de captación de energía, con el fin de garantizar la producción con intensidades lumínicas bajas (Valladares E Niinemets, 2008); sin embargo, en el caso de la cobertura de color verde, no ocurrió así. A pesar que esta cobertura presentó el mayor grado de opacidad, con lo cual se debería haber incrementado el área foliar, sucedió lo contrario, lo que permite deducir que, más que el sombreado causado por la cobertura, el área foliar fue afectada por el color.

Como consecuencia de la luz filtrada a través de los filmes de color, se apreciaron modificaciones morfoanatómicas en las plantas expuestas a las diferentes coberturas (Figura 2). En el análisis estadístico de la asignación de masa seca a los diferentes órganos en los que se seccionó la planta, se advirtieron diferencias altamente significativas en todos los casos $(P<0,01)$. Se observó que el menor porcentaje de acumulación de masa seca (AMS) en la raíz, se presentó en plantas expuestas a las coberturas amarilla y azul, mientras que la cobertura verde, registró el mayor valor. En la corona, se asignó menor cantidad de masa seca bajo la cobertura amarilla y en las plantas control y el mayor valor, se encontró bajo las coberturas azul y verde. Las plantas control mostraron la menor AMS en las hojas y la mayor se presentó en plantas colocadas bajo las coberturas de colores azul y verde. La cobertura de color rojo indujo la menor AMS en los estolones, mientras que el mayor porcentaje se presentó bajo el color verde. La cobertura transparente redujo la AMS en las flores y la de color verde, la aumentó. En cuanto a los frutos, se registró la menor AMS con la exposición de las plantas al color verde y, por el contrario, bajo la cobertura de color amarillo, se registró la mayor AMS.

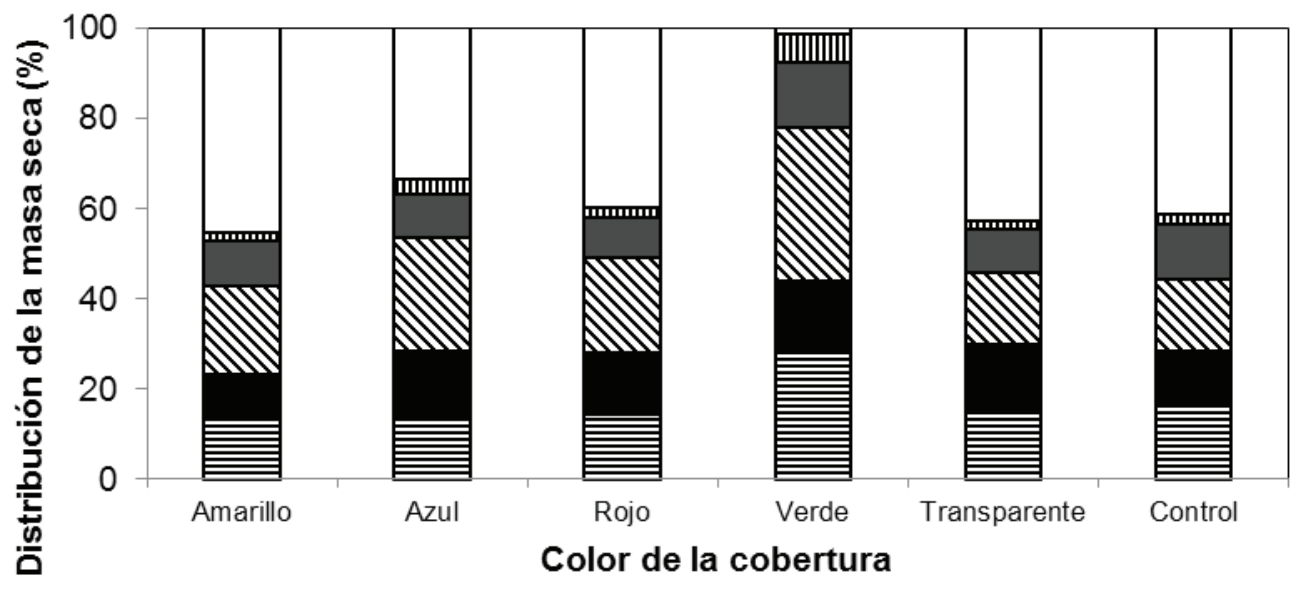

$\square$ Frutos mFlores $\square$ Estolones $\mathbf{v H o j a s} \mathbf{a}$ Corona 目Raíz

Figura 2. Distribución de la masa seca en plantas de fresa (Fragaria sp.), expuestas a coberturas de color. 
Las variaciones de la relación raíz:vástago (Tabla 2) son el resultado de la interacción de la calidad de la luz y el sombreado proporcionadas por las coberturas, debido a que existen parámetros anatómicos, morfológicos y fisiológicos de mayor o menor plasticidad, ante la variación de la calidad de luz (Terashima et al. 2005). Además, la teoría de la partición óptima supone que las plantas responden a las variaciones en la disponibilidad de recursos, mediante la partición preferencial de recursos entre los distintos órganos, de forma de optimizar así la captura de los mismos y maximizar, consecuentemente, la tasa de crecimiento (Hirose, 1987).

Así, el análisis de los patrones de asignación de biomasa resulta ser la mejor herramienta disponible para la investigación de las funciones prioritarias de las plantas (Weiner, 2004). Se asume que los organismos disponen de una cantidad limitada de recursos que deben ser divididos entre las principales funciones, generándose así relaciones de compromiso (tradeoffs), entre las mismas. Dado que los destinos son mutuamente excluyentes, los recursos destinados a una función o estructura, no están disponibles para otra (Reekie \& Bazzaz, 1987). En el presente estudio, la modificación más llamativa se halló en plantas colocadas bajo la cobertura de color verde, lo cual, pudo ser la consecuencia del sombreado causado por el polipropileno, pero también, se debe considerar que este material transmitió en mayor cantidad en un rango muy limitado del espectro (colores verde y amarillo: 510-540nm). Además, su trasmisión en los rangos del azul y del rojo estuvo alrededor de $20-30 \%$ (Figura 1). Este aspecto pudo limitar la captura de fotones, puesto que en estas franjas, se encuentra la mayor actividad de absorción de las clorofilas (Manrique, 2003). También, es conocido que el desempeño fotosintético depende de características bioquímicas y fitoquímicas, como la actividad de la enzima Rubisco y otras enzimas del ciclo $\mathrm{C}_{3}$, el contenido de clorofila a, y la eficiencia de los fotosistemas I y II y, en consecuencia, la disponibilidad de los productos ATP y NADPH (Chen et al. 1999). Por lo tanto, en el presente estudio, las plantas colocadas bajo la cobertura de color verde presentaron la menor AMS total en la planta, menor área foliar, menor peso específico de las hojas, en relación con los demás tratamientos, pero el mayor valor de la relación raíz:vástago.

Estos resultados indican que serían necesarios más estudios bajo diferentes intensidades de luz y de bandas de color específicas. En comunidades vegetales en campo, la distribución de luz no es uniforme, ya que presenta menor intensidad en la parte baja del perfil de las plantas. Además, existen diferentes bandas de luz dentro del perfil vegetal con variaciones desde la parte superior hasta la inferior, por tanto, es posible que las hojas inferiores reflejen más luz en el rango del verde que afecta la estructura y la función de las hojas vecinas. En este caso, los resultados reportados en el presente estudio tienen implicaciones agronómicas en relación de las densidades apropiadas de plantas.

Con vista en la importancia del cultivo de fresa y otros cultivos, en el departamento de Boyacá y otras ecozonas similares de Colombia, se deben iniciar investigaciones estratégicas agronómicas, apoyadas en estudios eco-fisiológicos, los cuales, serían de gran importancia para el desarrollo agrícola nacional.

Fluorescencia de la clorofila a: El análisis de variancia arrojó diferencias altamente significativas para la $\mathrm{F}_{\mathrm{o}}$ entre los tratamientos $(\mathrm{P}<0,01)$ (Tabla 3$)$. Además, se registró un coeficiente de variación de 28,91\%. La diferencia se expresó, básicamente, entre las plantas que se desarrollaron bajo la cobertura amarilla y las plantas control sin cobertura, en donde la primera indujo un valor de $\mathrm{F}_{\mathrm{o}} 89,32 \%$ mayor que en las plantas control.

Tabla 3. Valores promedio de parámetros de fluorescencia determinados en plantas de fresa (Fragaria sp.) expuestas a coberturas de color (datos por planta).

\begin{tabular}{|l|c|c|c|c|}
\hline \multirow{2}{*}{$\begin{array}{c}\text { Color de la } \\
\text { cobertura }\end{array}$} & Fluorescencia mínima & Fluorescencia terminal & Fluorescencia variable & Fluorescencia máxima \\
\cline { 2 - 5 } & $\left(\mathrm{F}_{0}\right)$ & $\left(\mathrm{F}_{\mathrm{t}}\right)$ & $\left(\mathrm{F}_{\mathrm{v}}\right)$ & $\left(\mathrm{F}_{\mathrm{m}}\right)$ \\
\hline Amarillo & $644,33 \mathrm{a}$ & $646,00 \mathrm{a}$ & $1273,67 \mathrm{ab}$ & $1918,00 \mathrm{a}$ \\
\hline Azul & $406,33 \mathrm{ab}$ & $409,33 \mathrm{bc}$ & $1372,67 \mathrm{a}$ & $1779,00 \mathrm{a}$ \\
\hline Rojo & $443,67 \mathrm{ab}$ & $451,00 \mathrm{abc}$ & $1315,00 \mathrm{a}$ & $1758,67 \mathrm{a}$ \\
\hline Verde & $407,33 \mathrm{ab}$ & $411,00 \mathrm{abc}$ & $1362,33 \mathrm{a}$ & $1769,67 \mathrm{a}$ \\
\hline Transparente & $617,00 \mathrm{a}$ & $624,33 \mathrm{ab}$ & $977,33 \mathrm{bc}$ & $1594,33 \mathrm{ab}$ \\
\hline Control & $340,33 \mathrm{~b}$ & $344,67 \mathrm{c}$ & $938,67 \mathrm{c}$ & $1279,00 \mathrm{~b}$ \\
\hline
\end{tabular}


Además, se apreció un aumento en el valor promedio de $\mathrm{F}_{0}$ en plantas expuestas a las coberturas transparente y amarilla. Como ya se había mencionado, la cobertura amarilla presenta su máxima transmitancia muy cercana al valor registrado para la cobertura transparente, por sobre los 530nm, por tanto, las respuestas morfo-fisiológicas que se pudieran producir en las plantas, por parte del espectro en el rango del verde al rojo, serían idénticas en las plantas, bajo estas dos coberturas. Gonçalves et al. (2001) notaron que bajo plena iluminación, en relación con plantas sombreadas, el valor de $\mathrm{F}_{0}$ se reduce en Swietenia macrophylla, mientras que esta medida no se altera en Dipteryx odorata, bajo las mismas condiciones, lo cual, implica diferencias genéticas.

Este primer nivel $\left(\mathrm{F}_{0}\right)$ es un indicativo de la plena actividad de los centros de reacción en la clorofila y pigmentos asociados. Refleja la pérdida de una parte de la energía absorbida por las moléculas-antena antes que éstas hayan podido transferir la energía de excitación hacia los centros de reacción (Briantais et al. 1986). Este nivel corresponde a un estado, donde todos los aceptores primarios de electrones del PSII (Quinona A) están oxidados (con carga energética positiva y falta de electrones), por tanto, es independiente de los procesos fotoquímicos. El incremento en la $\mathrm{F}_{0} \mathrm{y}$ la reducción del valor de la relación $F_{v} / F_{m}$, representan alteraciones a nivel de los centros de reacción del fotosistema II, reducción tanto en la capacidad fotosintética reducida como en la eficiencia fotoquímica (Willits \& Peet, 2001), daños colaterales o deterioro en la maquinaria fotosintética de la hojas y, en consecuencia, reducida fijación de $\mathrm{CO}_{2}$ (Percival \& Henderson, 2003). De hecho, en un estudio realizado por Casierra-Posada $\mathcal{E}$ PeñaOlmos (2011), bajo las mismas características y condiciones, se encontró que bajo las coberturas amarilla y transparente, el contenido de clorofila total fue bajo, en comparación con las coberturas de los demás colores, mientras que la relación carotenos / clorofila total fue alta, con lo cual, se pude inferir, que la alta cantidad de los carotenos podría tener una función protectora, puesto que estos compuestos (carotenos y xantofilas) presentan en las plantas una función doble, como pigmentos accesorios en la captación de energía lumínica y como moléculas capaces de disipar la energía de excitación excedente en forma de calor, evitando daños importantes, llamados fitoinhibción (Manrique, 2003; Demmig-Adams E Adams, 1992).

El valor registrado para la $F_{v}$ mostró diferencias altamente significativas $(P<0,01)$, entre el control y las plantas expuestas a las demás coberturas, con excepción de la cobertura transparente, con un coeficiente de variación de 17,20\%. Las plantas colocadas bajo las coberturas amarilla, azul, roja y verde presentaron valores de $F_{v}, 35,68 ; 46,23 ; 40,09$ y $45,13 \%$ más elevados que las plantas que crecieron sin cobertura (Tabla 3).
La respuesta más importante de la planta, en el caso de la fluorescencia determinada en hojas adaptadas a la oscuridad, es la $F_{v}$ (Vieira et al. 2010). Mientras mayor es el valor de la $F_{v}$ mayor será la capacidad del PSII para realizar la fotoquímica primaria y, en consecuencia, mayor será la capacidad de asimilar de $\mathrm{CO}_{2}$, en los procesos bioquímicos de la fotosíntesis (Baker E Rosenqvist, 2004), lo cual, no se vio reflejado en los resultados del presente trabajo, puesto que el valor de $F_{v}$, en todos los tratamientos, fue superior al de las plantas control sin cobertura; no obstante, estas últimas presentaron mayor producción de peso seco total, acorde con la tabla 2, lo que pudo ser la consecuencia de una mayor capacidad de disipación de la energía capturada, por el PSII, en forma de calor (Non-photochemical quenching).

Alternativamente, con menos área foliar por planta, pero con hojas más pesadas (peso específico alto, debido a mayor cantidad de componentes fotosintéticos y bioquímicos por unidad de área foliar (Tabla 2), la producción superior de biomasa en plantas control fue posible, debido a una mayor tasa de fijación de $\mathrm{CO}_{2}$ por unidad de área foliar. También, es posible que la densidad estomática (número por área foliar) y, como resultante, la conductancia foliar de difusión de $\mathrm{CO}_{2}$, hayan sido mayores en plantas sin cobertura. Es conocido que la tasa de fotosíntesis es positivamente asociada con la conductancia estomática, como por ejemplo, en el cultivo de yuca (El-Sharkawy, 2007). Factores relacionados con la anatomía foliar, características estomáticas y fisiológicas, como la tasa de fotosíntesis y bioquímicas, como cantidad y actividad de enzimas afectadas por la intensidad y calidad de luz, merecen más estudios en el futuro cercano.

Las lecturas registradas para la $\mathrm{F}_{\mathrm{m}}$ mostraron que hubo diferencia altamente significativa $(P<0,01)$, entre las plantas control y las aquellas expuestas a las demás coberturas, con excepción de las que crecieron bajo la cobertura transparente. Adicionalmente, se halló un coeficiente de variación de $14,95 \%$. Las plantas que se desarrollaron bajo las coberturas amarilla, azul, roja y verde presentaron valores de $\mathrm{F}_{\mathrm{m}} 49,96$; 39,$09 ; 37,50$ y $38,36 \%$ más elevados, que las plantas control sin cobertura (Tabla 3 ).

Se debe considerar que en este estudio, se utilizó un pulso actínico de luz $25 \mu \mathrm{mol} \mathrm{m}^{-2} \mathrm{~s}^{-1}$, con miras a lograr el valor de $\mathrm{F}_{\mathrm{m}}$, por tanto, al aumentar la cantidad de luz, tiene lugar una conversión progresiva de violaxantina en zeaxantina y un incremento en la concentración relativa de las formas deepoxidadas del ciclo de las xantofilas, frente a las más epoxidadas; por ende, existe un incremento gradual del estado de deepoxidación (DPS) del ciclo de las xantofilas, que se correlaciona con la disminución de la eficiencia fotoquímica del PSII, medida mediante fluorescencia de la clorofila a (Man- 
rique, 2003). El valor de $F_{m}$ fue superior en todos los tratamientos, en relación con el obtenido en las plantas control, lo que sería un índice de disipación de la energía capturada, en forma de florescencia, lo que impactó negativamente la producción de masa seca, mostrada en la tabla 2. Este resultado, se puede corroborar con el reporte de Casierra-Posada et al. (2011), quienes utilizaron la información colectada en el presente estudio, para realizar un análisis del crecimiento en las plantas y encontraron que la tasa de asimilación neta (TAN: la tasa de producción de biomasa total por área foliar) se redujo en las plantas expuestas a todas las coberturas de color, en relación con las plantas control, con excepción de la cobertura transparente. Estos hallazgos muestran que la tasa de fotosíntesis neta basada en área foliar $\left(P_{N}\right)$ fue afectada negativamente por las diferentes coberturas de color.

El ciclo de las xantofilas es un proceso flexible, que responde a las condiciones lumínicas, como fluctuaciones diurnas, insolaciones puntuales o transiciones sol-sombra o los llamados 'sunflecks', dentro del perfil vegetal (Demmig-Adams E Adams, 1992). En el ambiente natural, los vegetales están sometidos a la acción de estos y otros factores ambientales, que modulan su respuesta en conjunto; sin embargo, las diferentes especies responden de forma muy diferente a las fluctuaciones. Estas diferencias fisiológicas entre especies son las que contribuyen, de forma definitiva, a las diferencias funcionales y adaptivas entre ecosistemas (Manrique, 2003).
Se apreció diferencia altamente significativa en los valores registrados para la eficiencia fotoquímica del PSII $\left(\mathrm{F}_{\mathrm{v}} / \mathrm{F}_{\mathrm{m}}\right)$ $(P<0,01)$, con un coeficiente de variación de 9,28\%. Esta diferencia se manifestó en especial, en el valor registrado en las plantas control sin cobertura y las que crecieron bajo la cobertura transparente, las cuales, exhibieron un valor de $\mathrm{F}_{\mathrm{v}} / \mathrm{F}_{\mathrm{m}} 16,57 \%$ menor que las plantas del tratamiento control (Figura 3).

$\mathrm{F}_{\mathrm{v}} / \mathrm{F}_{\mathrm{m}}$ se correlaciona bien con el rendimiento cuántico fotosintético, medido como la producción de $\mathrm{O}_{2} \mathrm{O}$ el consumo de $\mathrm{CO}_{2}$ a bajas irradiancias. Una reducción en $\mathrm{F}_{\mathrm{v}} / \mathrm{F}_{\mathrm{m}}$ puede ser generada por una reducción en $\mathrm{F}_{\mathrm{m}} \mathrm{y} / \mathrm{o}$ por un incremento en $\mathrm{F}_{0}$, como sucedió en el presente trabajo, en el cual, la reducción en el valor de $\mathrm{F}_{\mathrm{v}} / \mathrm{F}_{\mathrm{m}}$ en las plantas expuestas a las coberturas amarilla y transparente, con respecto a las plantas control, se debió al aumento en el valor de $\mathrm{F}_{0}$, y no a la reducción del valor de $F_{m}$. Valores de $F_{v} / F_{m}$ en el rango de 0,78 a 0,85 , se asocian con plantas sanas y no estresadas (Percival, 2004). La reducción en el valor de la relación $F_{v} /$ $\mathrm{F}_{\mathrm{m}}$ encontrado en el presente estudio en plantas que crecieron bajo las coberturas de colores amarillo y transparente, posiblemente, se atribuya a un estado alto de reducción del primer receptor de electrones (el pool de $Q_{A}$ ), que conlleva a una variación en la tasa de transporte de electrones (Baker $\mathcal{E}$ Rosenqvist, 2004). Por otro lado, los valores de la relación $\mathrm{F}_{\mathrm{v}} / \mathrm{F}_{\mathrm{m}}$ encontrados en plantas expuestas a las coberturas de

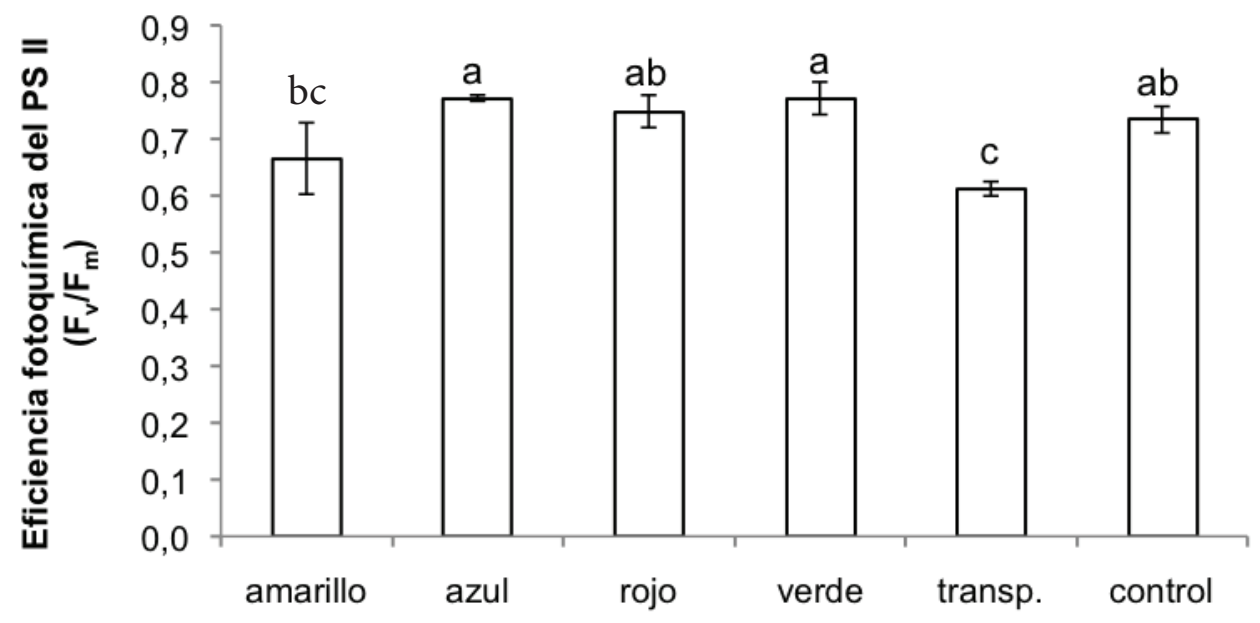

Color de la cobertura

Figura 3. Eficiencia fotoquímica del PS II determinada en plantas de fresa (Fragaria sp.), expuestas a coberturas de color. 
color azul, rojo y verde, posiblemente, se encuentren relacionados con la eficiencia de los mecanismos fotoprotectores, que pueden modular la probabilidad de fotodaño, mediante ajustes morfológicos, cambios en el tamaño de la antena y activación de rutas alternas de transporte de electrones (Niyogi, 1999).

Por último, se encontró diferencia estadísticamente significativa en las mediciones de $\mathrm{F}_{\mathrm{t}}(\mathrm{P}<0,01)$. Las plantas expuestas a todas las coberturas presentaron valores de $F_{t}$ superiores a las plantas control (Tabla 3). Al respecto, Bacarin $\&$ Mosquim (2002), mencionan que el aumento del valor de este parámetro, normalmente viene acompañado de un incremento en la tasa de fotosíntesis neta $\left(P_{N}\right)$, como resultante de mayor asimilación de $\mathrm{CO}_{2}$, con utilización de ATP y de NADPH en la fase bioquímica, los cuales, se produjeron en la fase fotoquímica; sin embargo, por el contrario al reporte de estos autores, en este estudio, los valores registrados para el peso seco total en plantas, bajo todas las coberturas, disminuyó (Tabla 2) y se puede interpretar como una reducción en la tasa de asimilación, lo cual, fue corroborado por Casierra-Posada et al. (2011) quienes, con las mismas plantas del presente estudio, hallaron que la tasa de asimilación neta se redujo en las plantas expuestas a todas las coberturas de color, en relación con las del control, excepto en aquellas bajo la cobertura transparente.

Conflicto de intereses: El manuscrito fue preparado y revisado con la participación de todos los autores, quienes declaramos que no existe ningún conflicto de intereses que ponga en riesgo la validez de los resultados presentados. Financiación: Este estudio se desarrolló con el apoyo de la Dirección de Investigaciones (DIN) de la Universidad Pedagógica y Tecnológica de Colombia (UPTC), en el programa Jóvenes Investigadores UPTC, con el soporte del grupo de investigación Ecofisiología Vegetal, adscrito al programa de Ingeniería Agronómica de la Facultad de Ciencias Agropecuarias de la UPTC.

\section{BIBLIOGRAFÍA}

1. BACARIN, M.A.; MOSQUIM, P.R. 2002. Cinética de emissão de fluorescência das clorofilas de dois genótipos de feijoeiro. Ciência e Agrotecnologia, Lavras. 26(4):705-710.

2. BAKER, N.R.; ROSENQVIST, E. 2004. Applications of chlorophyll fluorescence can improve crop production strategies: an examination of future possibilities. J. Exp. Bot. 55(403):1607-1621.
3. BRADBURNE, J.A.; KASPERBAUER, M.J.; MATHIS, J.N. 1989. Reflected Far-Red light effects on cholorophyll and light-harvesting chlorophyll protein (LHCII) contents under field conditions. Plant Physiol. 91(3):800-803.

4. BRIANTAIS, J.M.; VERNOTTE, C.; KRAUSE, G.H.; WEIS, E. 1986. Chlorophyll fluorescence of higher plant chloroplasts and leaves. In: Govindjee, J.A.; Fork, D.C., Eds. Light emission by plants and photosynthetic bacteria. Academic Press, New York. p.539-577.

5. CASIERRA-POSADA, F.; ROJAS B., J.F. 2009. Efecto de la exposición del semillero a coberturas de colores sobre el desarrollo y productividad del brócoli (Brassica oleracea var. italica). Agr. Col. 27(1):49-55.

6. CASIERRA-POSADA, F; PEÑA-OLMOS, J.E. 2011. Contenido de pigmentos en hojas de fresa (Fragaria sp) expuestas a diferente calidad espectral. Agr. Col. (en prensa).

7. CASIERRA-POSADA, F.; PEÑA-OLMOS, J.E.; ULRICHS, C. 2011. Análisis básico del crecimiento en plantas de fresa (Fragaria sp.) expuestas a diferente calidad de luz. Agr. Col. (en prensa).

8. CHEN, K.; HU, G.; KEUTGEN, K. 1999. Effects of $\mathrm{NaCl}$ and $\mathrm{CO}_{2}$ enrichment on pepino (Solanum muricatum Ait) II. Leaf photosynthetic properties and gas exchange. Sci. Hort. 81:43-56.

9. DECOTEAU, D.R. 2008. The emergence and early development of colored reflective plastic mulch technology in agriculture. En: Stevens, C.; Khan, V.A. (eds). Recent advances in agriculture. Research Signpost. Kerala, India. p.1-17.

10. DEMMIG-ADAMS, B.; ADAMS, III W.W. 1992. Photochemical and other responses of plants to high light stress. Ann. Rev. Plant Physiol. Plant Mol. Biol. 43:599-626.

11. EL-SHARKAWY, M.A. 2007. Physiological characteristics of cassava tolerance to prolonged drought in the tropics: Implications for breeding cultivars adapted to seasonally dry and semiarid environments. Braz. J. Plant Physiol. 19(4):257-286.

12. FOLTA, K.M.; MARUHNICH, S.A. 2007. Green light: a signal to slow down or stop. J. Exp. Bot. 58:3099-3111. 
13. FUKUDA, N.; FUJITAN, M.; OHTA, Y.; SASE, S.; NISHIMURA, S.; EZURA, H. 2008. Directional blue light irradiation triggers epidermal cell elongation of abaxial side resulting in inhibition of leaf epinasty in geranium under red light condition. Sci. Hort. 115:176-182.

14. GONÇALVES, J.F. de C.; MARENCO, R.A.; VIEIRA, G. 2001. Concentration of photosynthetic pigments and chlorophyll fluorescence of Mahogany and Tonka bean under two light environments. Rev. Bras. Fisiol. Veg. 13(2):149-157.

15. GONZÁleZ M., S.; PERALES V, H.; SALCEDO A., M.O. 2008. La fluorescencia de la clorofila a como herramienta en la investigación de efectos tóxicos en el aparato fotosintético de plantas y algas. Rev. Educación Bioquímica. 27(4):119-129.

16. HALIAPAS, S.; YUPSANIS, T.A.; SYROS, T.D.; KOFIDIS, G.; ECONOMOU, A.S. 2008. Petunia $x$ hybrida during transition to flowering as affected by light intensity and quality treatments. Acta Physiol. Plant. 30:807-815.

17. HIROSE, T. 1987. A vegetative plant growth model: Adaptive significance of phenotypic plasticity in matter partitioning. Functional Ecol. 1:195-202.

18. IMAIZUMI, T.; TRAN, H.G.; SWARTZ, T.E.; BRIGGS, W.R.; KAY, S.A. 2003. FKF1 is essential for photoperiodicspecific light signalling in Arabidopsis. Nature. 426:302306.

19. JIAO, Y.; LAU, O.S.; DENG, X.W. 2007. Light-regulated transcriptional networks in higher plants. Nat. Rev. Genet. 8:217-230.

20. LOUGHRIN, J.H.; KASPERBAUER, M.J. 2002. Aroma of fresh strawberries is enhanced by ripening over red versus black mulch. J. Agric. Food Chem. 50:161-165.

21. MANRIQUE, E. 2003. Los pigmentos fotosintéticos, algo más que la captación de luz. Ecosistemas 12(1). Disponible desde internet en: http://rua.ua.es/dspace/ bitstream/10045/8958/1/ECO_12\%281\%29_08.pdf. (con acceso 22/07/2011).

22. MIRANDA, J.H.; WILLIAMS, R. 2007. Developmental influence of in vitro light quality and carbon dioxide on photochemical efficiency of PS II of strawberry leaves (Fragaria $x$ ananassa). J. Appl. Hort. 9(1):13-16.
23. MATHEWS, S. 2010. Evolutionary studies illuminate the structural-functional model of plant phytochromes. The Plant Cell. 22:4-16.

24. NIYOGI, K.K. 1999. Photoprotection revisited: Genetic and molecular approaches. Ann. Rev. Plant Physiol. Plant Molec. Bio. 50:333-359.

25. PERCIVAL, G.C. 2004. Evaluation of physiological tests as predictors of young tree establishment and growth. J. Arboric. 30(2):80-92.

26. PERCIVAL, G.C.; HENDERSON, A. 2003. An assessment of the freezing tolerance of urban trees using chlorophyll fluorescence. J. Hortic. Sci. Biotech. 78(2):254-260.

27. REEKIE, E.G.; BAZZAZ, F.A. 1987. Reproductive effort in plants: effect of reproduction on vegetative activity. Am. Naturalist. 129:907-919.

28. SHAHAK, Y.; GAL, E.; OFFIR, Y.; BEN-YAKIR, D. 2008. Photoselective Shade Netting Integrated with Greenhouse Technologies for Improved Performance of Vegetable and Ornamental Crops. Acta Hort. 797:75-80.

29. STRASSER, B.; SÁNCHEZ-LAMAS, M.; YANOVSKY, M.J.; CASAL, J.J.; CERDÁN, P.D. 2010. Arabidopsis thaliana life without phytochromes. Proc. Nal. Acad. Sci. U.S.A. 107:4776-4781.

30. TERASHIMA, I.; HANDA, Y.T.; TAZOE, Y.; VYAS, P.; YANO, S. 2005. Irradiance and phenotype: comparative ecodevelopment of sun and shade leaves in relation to photosynthetic $\mathrm{CO}_{2}$ diffusion. J. Exp. Bot. Phenothypic Plasticity and the Changing Environm. Special Issue. 57(2):343-354.

31. VALLADARES, F.; NIINEMETS, Ü. 2008. Shade Tolerance, a Key Plant Feature of Complex Nature and Consequences. Ann. Rev. Ecol. Evolution and Systematics. 39:237-257.

32. VIEIRA, D.A. de P.; PORTES, T. de A.; STACCIARINISERAPHIN, E; TEIXEIRA, J.B. 2010. Fluorescência e teores de clorofilas em abacaxizeiro cv. pérola submetido a diferentes concentrações de sulfato de amônio. Rev. Bras. Frutic. 32(2):360-368.

33. WEINER, J. 2004. Allocation, plasticity and allometry in plants. Perspectives in Plant Ecol., Evol. and Systematics. 6(4):207-215. 
34. WILLITS, D.H.; PEET, M.M. 2001. Using chlorophyll fluorescence to model leaf photosynthesis in greenhouse pepper and tomato. Acta Hortic. 507:311315.

Recibido: Julio 24 de 2011

Aceptado: Noviembre 2 de 2011 